\title{
Closing the circadian negative feedback loop: FRQ-dependent clearance of WC-1 from the nucleus
}

\author{
Christian I. Hong, ${ }^{1}$ Peter Ruoff, ${ }^{2}$ Jennifer J. Loros, ${ }^{1,3}$ and Jay C. Dunlap ${ }^{1,4}$ \\ ${ }^{1}$ Department of Genetics, Dartmouth Medical School, Hanover, New Hampshire 03755, USA; ${ }^{2}$ Department of Mathematics \\ and Natural Science, University of Stavanger, N-4036 Stavanger, Norway; ${ }^{3}$ Department of Biochemistry, Dartmouth \\ Medical School, Hanover, New Hampshire 03755, USA
}

\begin{abstract}
In Neurospora crassa, a transcription factor, WCC, activates the transcription of frq. FRQ forms homodimers as well as complexes with an RNA helicase, FRH, and the WCC, and translocates into the nucleus to inactivate the WCC, closing the time-delayed negative feedback loop. The detailed mechanism for closing this loop, however, remains incompletely understood. In particular within the nucleus, the low amount of FRQ compared with that of WC-1 creates a conundrum: How can the nuclear FRQ inactivate the larger amount of WCC? One possibility is that FRQ might function as a catalytic component in phosphorylation-dependent inhibition. However, in silico experiments reveal that stoichiometric noncatalytic binding and inhibition can generate a robust oscillator, even when nuclear FRQ levels are substantially lower than nuclear WCC, so long as there is FRQ-dependent clearance of WC-1 from the nucleus. Based on this model, we can predict and now demonstrate that WC-1 stability cycles, that WC-1 is stable in the absence of FRQ, and that physical binding between FRQ and WCC is essential for closure of the negative feedback loop. Moreover, and consistent with a noncatalytic clearance-based model for inhibition, appreciable amounts of the nuclear FRQ:WCC complex accumulate at some times of day, comprising as much as $10 \%$ of the nuclear WC-1.
\end{abstract}

[Keywords: Circadian; negative feedback; FRQ; WC-1]

Supplemental material is available at http://www.genesdev.org.

Received June 19, 2008; revised version accepted September 10, 2008.

A network of complex dynamic processes underlies circadian rhythms. Events ranging from the activation of clock genes to the modifications of clock proteins involve regulatory control mechanisms as a function of circadian time $(\mathrm{CT})$. The ensemble of processes creates a robust oscillatory system with a period of $22 \mathrm{~h}$ in Neurospora crassa. The mechanism of this robust oscillator is largely based on a time-delayed negative feedback loop where an activator (positive arm) activates an inhibitor (negative arm) that will repress its own activator (Dunlap 1999; Liu and Bell-Pedersen 2006). A heterodimeric transcription factor, WCC, is the positive arm, and FRQ complexed with FRH is the negative arm. FRQ cycles in abundance, undergoes extensive and progressive phosphorylation (Garceau et al. 1997), and is assumed to carry out various functions at different CTs as a result of these post-translational modifications. The level of WC-1 cycles anti-phasic to that of FRQ (Lee et al. 2000) and is low in the frq-null mutant $\left(f_{r q} q^{10}\right)$ (Lee et al. 2000). The

\footnotetext{
${ }^{4}$ Corresponding author.

E-MAIL Jay.C.Dunlap@Dartmouth.edu; FAX (603) 650-1233.

Article published online ahead of print. Article and publication date are online at http://www.genesdev.org/cgi/doi/10.1101/gad.1706908.
}

daily increase in WC-1 abundance is correlated with the appearance of the hyperphosphorylated, or mature, form of FRQ, reflecting a positive role of FRQ in augmenting the level of WC-1 (Lee et al. 2000; Schafmeier et al. 2006). Previously, it was shown that FRQ physically interacts with the WCC (Cheng et al. 2001a, 2002; Denault et al. 2001). Hence, it was assumed that the physical interactions of FRQ with the WCC are at the core of inactivating the WCC, although the detailed dynamics of the negative feedback mechanism are yet to be uncovered.

Recently, Schafmeier et al. (2005) suggested that stoichiometric physical interactions between FRQ and the WCC are not sufficient to close the feedback loop. First of all, weak or transient interactions between FRQ and WCC were reported. Second, because most of the FRQ resides in the cytoplasm while most of the WC-1 is in the nucleus, the abundance of nuclear WC- $1\left(\mathrm{WC}-1_{\mathrm{n}}\right)$ is far greater than the nuclear FRQ $\left(\mathrm{FRQ}_{\mathrm{n}}\right)$. This compartmentalized abundance of activator (WC-1) and inhibitor (FRQ) creates a conundrum: How does FRQ inhibit the WCC? As Schafmeier et al. (2005) suggested, a sensible way to decipher this problem is to suggest that FRQ acts as an enzyme in a catalytic role to inactivate WCC via FRQ-promoted phosphorylation. The catalytic activity of FRQ, however, is yet to be demonstrated. 
Within recent years, the use of computational modeling and systems approaches to inform experimentation has begun to impact the analysis of circadian rhythms in fungi, plants, and animals (Gallego et al. 2006; Locke et al. 2006; Hong et al. 2007; Ueda 2007; Akman et al. 2008). Recently, we also developed a mathematical model for the Neurospora clock and used this to test different possible closure mechanisms for the negative feedback loop (Hong et al. 2008). These model simulations provided several insights: First, it is possible for the 1:1 noncatalytic stoichiometric binding of FRQ to WC-1 to provide a plausible mechanism for closure of the negative feedback loop, even when the level of FRQ is substantially lower than that of WC-1 in the nucleus, so long as there is FRQ-dependent clearance of WC-1 from the nucleus. In this model, WC-1 disappears rapidly together with FRQ as a complex. The model makes the obvious but untested prediction that nuclear WC-1 levels will normally cycle but that WC- $1_{n}$ will be stable in the absence of FRQ. Also, physical binding between FRQ and the WCC will be important for the FRQ-dependent clearance of WC-1, and the complex of FRQ:WCC is predicted to rise to appreciable levels and then cycle in amount within the nucleus. Such a relatively stable interaction would not be expected if there is only a weak or transient interaction as suggested from previous reports.

The experimental data reported here support all of these predictions and, moreover, reveal a daily cycle in the stability of total WC-1 that was not predicted from the model. Specifically, turnover of WC-1 is correlated with the appearance of hypophosphorylated FRQ. Taken together, the data support a model in which FRQ makes a complex with the WCC and clears it from the nucleus, thereby preventing it from binding to DNA to activate transcription of frq. This FRQ-dependent clearance of WC-1 from the nucleus contributes to closing of the negative feedback loop.

\section{Results}

FRQ modulates the stability of WC-1 as a function of $C T$

Prior to testing FRQ-dependent clearance of WC-1 from the nucleus, we wanted to determine the half-lives of both proteins as a function of CT. Both FRQ and WC-1 cycle in amount and go through protein modifications that may affect their stability. Samples were treated with cycloheximide (CHX) at the CT of interest and followed for $12 \mathrm{~h}$ in $\mathrm{CHX}$ with 4 -h resolution (Fig. 1A). WC-2 is much more abundant than FRQ or WC-1 and appears to be stable regardless of CT (Cheng et al. 2001b; Denault et al. 2001; data not shown). FRQ is relatively unstable throughout the circadian day, and the half-lives of FRQ that we calculate are comparable with previously measured values ( 2.5-4 h) (He et al. 2003; Ruoff et al. 2005). The overall stability of WC-1 is greater compared with FRQ but also changes as a function of CT (Fig. 1B). WC-1 is relatively less stable at CT3-5 and remarkably stable at CT14. This was an unexpected finding-not predicted

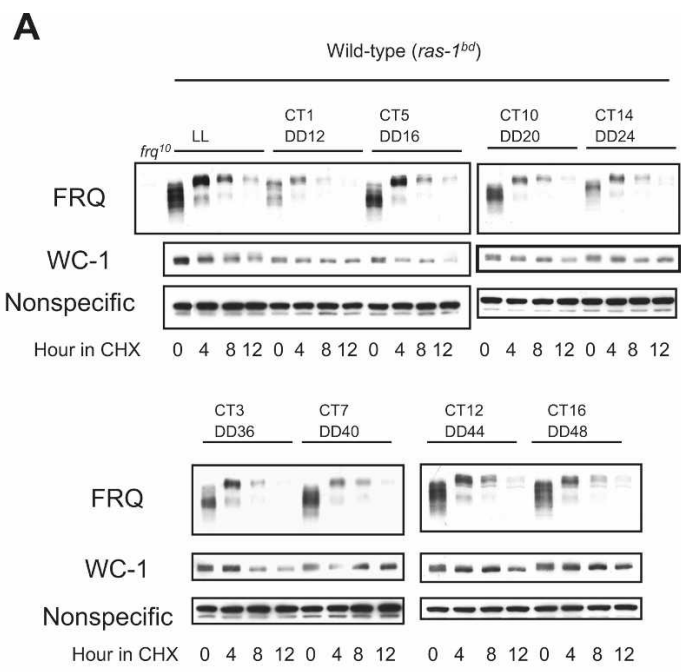

B

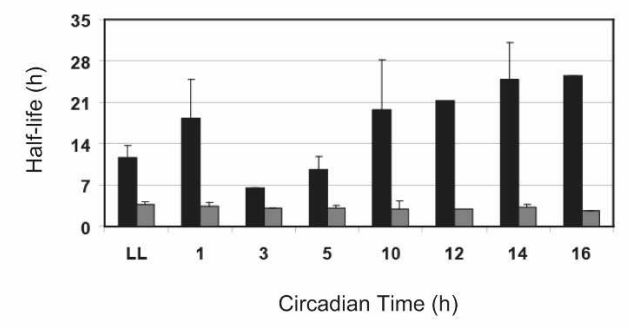

DWC-1 घFRQ

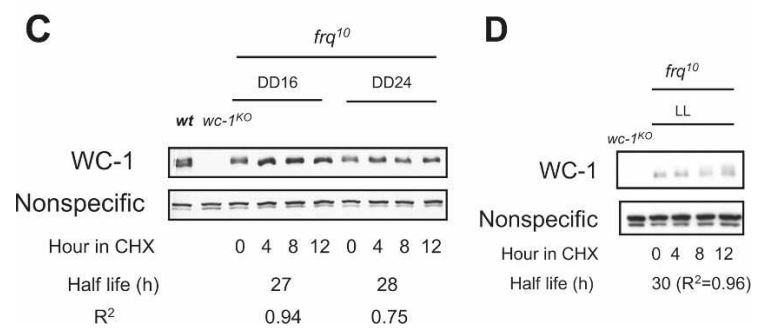

Figure 1. The stability of WC-1 changes as a function of CT. (A) The half-lives of both FRQ and WC-1 were measured as a function of CT. Cultures were grown as described in the Materials and Methods, CHX was added at the indicated $\mathrm{CT}$, and samples were harvested over the subsequent $12 \mathrm{~h}$. FRQ is phosphorylated and degraded in a similar fashion at all times. WC-1 shows different stability profiles depending on the CT. A nonspecific band is shown as a loading control. (B) Half lives of FRQ and WC-1 were determined through densitometric analysis of blots such as those shown in $A$, plotting the change in optical density of each band as a function of time. Error bars represent ${ }_{ \pm} \mathrm{SD}(n=3)$. Two-way ANOVA indicates that there is no significant difference of half-life in FRQ $(P=1.0)$, but there is significant difference of half-life in WC-1 $(P=0.004)$ at different time points. $(C)$ WC-1 is relatively stable in the absence of FRQ. Cultures were grown and processed as in $A$ and half life determined as in $B$. $(D)$ FRQ-independent phosphorylation and turnover of WC-1. WC-1 in $f r q^{10}$ is more stable than WC-1 in the wild-type background in LL (shown in $A, B$ ). Linear ranges of FRQ and WC-1 are measured and indicated in the Supplemental Material (Supplemental Fig. S1). $\mathrm{R}^{2}$ values from the linear regressions of densitometry versus time provide an estimate of precision for the half-life determinations. 
from the mathematical model - that could influence the kinetics of negative feedback.

Previously, it was reported that the amount of WC-1 is low in the absence of FRQ, and that the hyperphosphorylated (or mature) form of FRQ is correlated with and promotes accumulation of WC-1 (Lee et al. 2000; Schafmeier et al. 2006). Hence, it is possible that the innate stability of WC-1 is constant, but the post-transcriptional activation of WC-1 by the mature FRQ may create what looks like more stable WC-1. In other words, our observation may be a mere reflection of previous results. In order to address this, we measured the half-life of WC-1 in the frq-null mutant $\left(f r q^{10}\right)$ (Fig. 1C). WC-1 is remarkably stable in the absence of FRQ at two different times in constant darkness (DD16 and DD24) even though the endogenous level of WC-1 is lower than in the wild type as reported previously (Fig. 1C). Another noteworthy observation is that the mechanisms regulating degradation of WC-1 in constant light (LL) appear in part distinct from those regulating its degradation in DD. As previously observed, WC-1 goes through phosphorylation before degradation in LL (Fig. 1D; Talora et al. 1999|. Furthermore, this appears to be a FRQ-independent phenomenon as we observe that phosphorylation of WC-1 is robust in LL in the $f r q^{10}$ mutant. Intriguingly, WC-1 is more stable in LL in the $f r q^{10}$ strain than in wild type (Fig. 1, cf. A and D).

To further investigate the stability changes of WC-1 in correlation with FRQ, we used an inducible system where we can artificially control the expression of FRQ in the $f r q^{10}$ background $\left(r a s-1^{b d}\right.$; $\left.f r q^{10} ; q a-2 p-f r q\right)$ (Aronson et al. 1994). FRQ is induced and goes through extensive phosphorylation over time upon addition of the inducer, quinic acid (QA) (Fig. 2A). WC-1, on the other hand, displays peculiar dynamics. From its initial conditions, the level of WC-1 is decreased in correlation with an intermediate phosphoisoform of FRQ (Fig. 2A). A hyperphosphorylated form of FRQ promotes WC-1 accumulation at later times of induction, as previously shown (Lee et al. 2000; Schafmeier et al. 2006). In the absence of the inducer, there is no FRQ and WC-1 randomly fluctuates (Fig. 2A). These intriguing results, in particular the transient FRQ-induced turnover of WC-1, were actually previously observed but not noted, as that study only focused on the positive role of FRQ in augmenting the level of WC-1 (Fig. 2A; see also Fig. 2A in Lee et al. 2000). In order to confirm the disappearance of WC-1 in correlation with an intermediate phosphoisoform of FRQ, we performed a QA pulse followed by a wash and a second QA pulse. We observe that WC-1 decreases upon the initial induction of FRQ (DD2, DD4, and DD6), and it decreases again with reinduction of FRQ (DD27 and DD29) (Fig. 2B). Furthermore, we measured the half-lives of WC-1 starting after different durations of induction by QA (Fig. 2C). A 4-h induction leads to intermediate phosphoisoforms of FRQ whereas a 12-h induction leads to hyperphosphoisoforms. We treated samples with CHX after either 4- or 12-h induction by QA, and followed the stability of both FRQ and WC-1 for $12 \mathrm{~h}$ in CHX. We observe that WC-1 is rela-
A

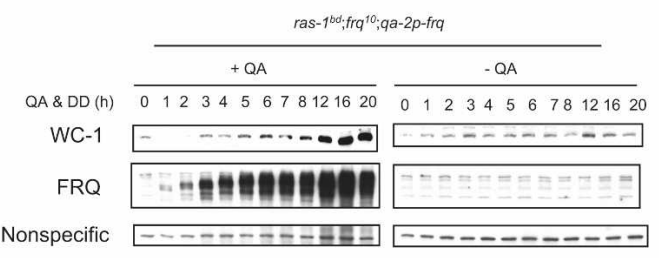

B

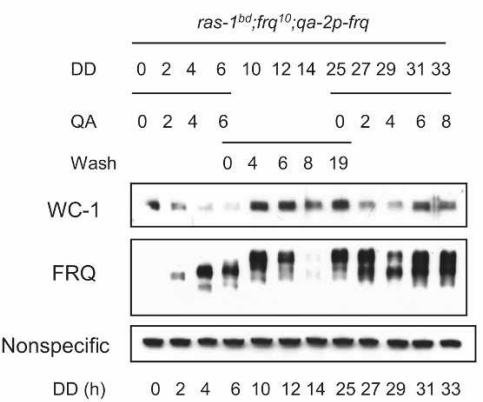

C
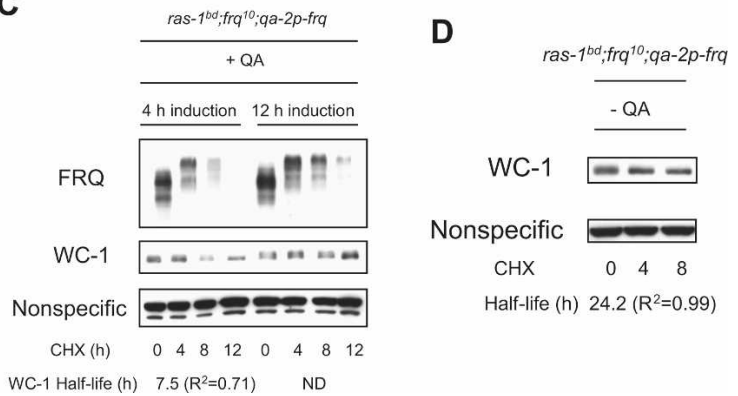

Figure 2. An intermediate phosphoisoform of FRQ is correlated with turnover of WC-1. $(A)$ Cultures were grown and QA added at time 0 to induce synthesis of FRQ as described in Materials and Methods. FRQ is induced and goes through multiple phosphorylations over time (middle left panel), whereas WC-1 transiently decreases (DD1 and DD2), and then accumulates (top left panel). (Right panels) In the absence of QA, there is no detectable FRQ, and WC-1 shows random fluctuations over time. Bands appearing in the right middle panel are nonspecific background. $(B)$ Induction of FRQ by QA followed by wash and reinduction. FRQ is induced for $6 \mathrm{~h}$ when QA is introduced, and WC-1 first decreases in correlation with intermediate phosphoisoform of FRQ and then increases as hyperphosphorylated FRQ accumulates. When the medium is changed to remove the QA, FRQ and WC-1 are stable, but reintroduction of QA at DD25 elicits de novo synthesis of FRQ and another transient reduction of WC-1. Two-way ANOVA indicates that there are no significant differences in the amount of the nonspecific band at different time points $(P=0.99)$, whereas there is a significant difference in the amount of WC-1 at different time points $(P=0.001)$. Absence of FRQ at DD14 is due to a gel artefact. The abundance of WC-1 increases in correlation with hyperphosphorylated FRQ as shown previously (Lee et al. 2000; Schafmeier et al. 2006). (C) FRQ was induced either $4 \mathrm{~h}$ or $12 \mathrm{~h}$ by QA before addition of CHX. Samples were harvested over 12 $\mathrm{h}$ in CHX with $4 \mathrm{~h}$ resolution. WC-1 is less stable when CHX is added to cultures containing the intermediate phosphoisoform of FRQ starting at $4 \mathrm{~h}$ after induction. Half lives were calculated as in Figure 1. $(D)$ WC-1 is relatively stable in the absence of FRQ (no QA). 
tively less stable in correlation with the intermediate phosphoisoform of FRQ (Fig. 2C), which reflects what we observed at CT5 in the wild type (Fig. 1A). WC-1 seems rather stable in the absence of FRQ with no inducer (Fig. 2D), reflecting its behavior in $\mathrm{frq}^{10}$ (Fig. 1C). Accumulating data suggest that FRQ modulates the stability of total WC-1 as a function of CT. Therefore, FRQ cannot only promote the accumulation of WC-1 but also promotes its turnover, thereby actively modulating the stability of WC-1 as a function of CT.

\section{FRQ-dependent clearance of WC-1 from the nucleus}

This information describing the half-lives of both FRQ and WC-1 at different CTs provides a good perspective for testing a central hypothesis deriving from the mathematical model: FRQ-dependent clearance of WC-1 from the nucleus.

Intuitively, we thought that the clearance rate of WC-1 from the nucleus may be faster at CT5 than CT14 based on the results obtained with the total lysates (Fig. $1 \mathrm{~A}, \mathrm{~B})$. From the model, however, we predicted constant rapid clearance of both $F R Q_{n}$ and $W C-1_{n}$ from the nucleus regardless of CT (Hong et al. 2008). In order to test this (Fig. 3A), we treated samples with CHX at the indicated CTs and followed changes in total and nuclear FRQ and WC-1. As in Figure 1, samples from the total lysates show that WC- 1 is more stable at CT5 versus CT14, and the overall stability of WC-1 is greater than that of FRQ (Fig. 3A,B). In contrast, both $\mathrm{FRQ}_{\mathrm{n}}$ and WC$1_{n}$ are rapidly cleared from the nucleus after the CHX treatment regardless of the starting CTs (CT5 vs. CT14). Interestingly, densitometric analysis indicates that the clearance rates of $\mathrm{FRQ}_{\mathrm{n}}$ and $\mathrm{WC}-\mathrm{1}_{\mathrm{n}}$ from the nucleus are virtually identical, as qualitatively predicted from the model, which is consistent with their being cleared out as a complex (Fig. 3B). Lastly, both $\mathrm{FRQ}_{\mathrm{n}}$ and $\mathrm{WC}-\mathrm{1}_{\mathrm{n}}$ are significantly decreased in the nucleus after $8 \mathrm{~h}$ in CHX, whereas total FRQ and WC-1 are still abundant. These results reveal two things: (1) WC-1 appears not to be predominantly nuclear at all times at least under these experimental conditions, in contrast to conclusions from previous studies (Schafmeier et al. 2005); and (2) there may be degradation in the nucleus and/or export of $F R Q_{n}$ and $\mathrm{WC}-1_{\mathrm{n}}$ to the cytoplasm. The mathematical model had predicted that this clearance of $\mathrm{WC}-1_{\mathrm{n}}$ is a FRQdependent mechanism; a corollary is that WC-1 should be stable in the nucleus in the absence of FRQ. To test this hypothesis, we performed a similar experiment with a frq-null mutant $\left(\right.$ fr $\left.q^{10}\right)$, and found WC- $1_{n}$ to be stable in the nucleus in the absence of FRQ in CHX (Fig. 3C).

The physical interaction between FRQ and WC-1 is generally considered to be a key process for closing the negative feedback loop. Given this, mutants that alter this interaction might be expected to affect the rate of clearance of WC-1 from the nucleus. To test this, we used a strain $\left(\mathrm{fr}_{\mathrm{r}}{ }^{9}\right.$;Myc- $\left.f r q 1\right)$ in which the coiled-coil region of FRQ is deleted (Cheng et al. 2001a). In such strains, FRQ fails either to dimerize or to interact with WC-1. In the mutant, FRQ accumulates and is phosphor-
A

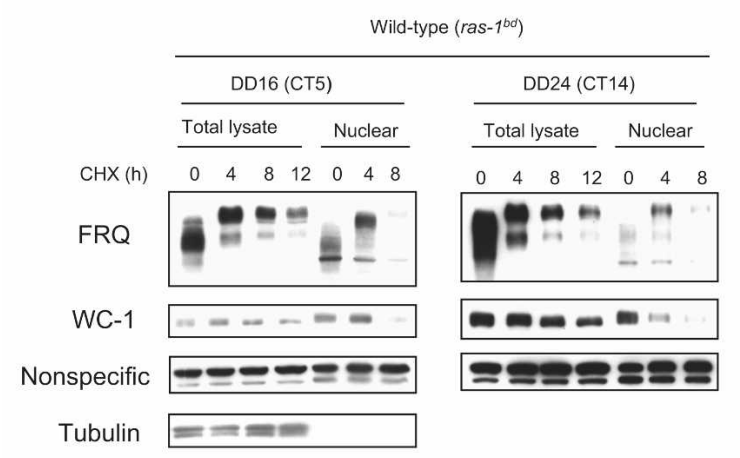

B

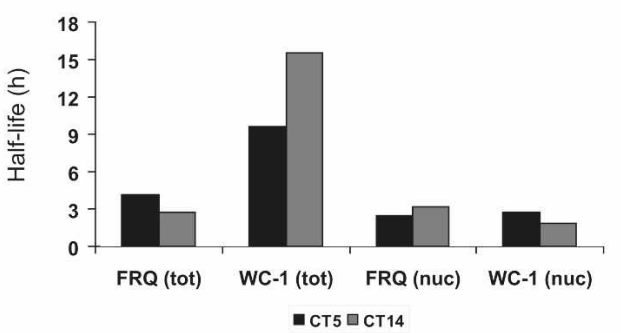

C

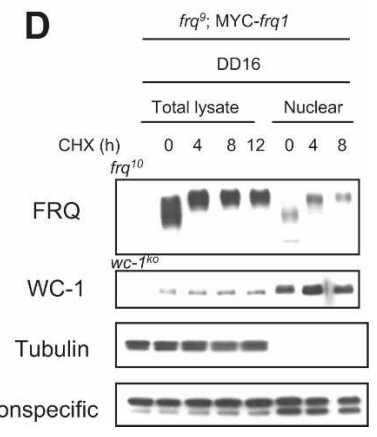

Figure 3. FRQ-dependent clearance of WC-1 from the nucleus. (A) Total and nuclear profiles of both FRQ and WC-1 were followed after addition of CHX at two different times. Although total WC-1 slowly turns over (see also Fig. 1A), both FRQ and WC- 1 are rapidly cleared from the nucleus by hour 8 in CHX regardless of initial CT. Tubulin is cytoplasmic and is used as a control to validate the quality of the nuclear preparations. $(B)$ The densitometric analysis shown in $A$ indicates that the clearance rates of $F R Q_{n}$ and $W C-1_{n}$ from the nucleus are similar and distinct from the half-life of total WC-1. Two-way ANOVA indicates that the half-life of total FRQ is significantly different from that of total WC-1 at both CT5 $(P=0.0005)$ and CT14 $(P<0.0001)$. Moreover, there is a significant difference between the half-lives of total WC-1 versus both nuclear FRQ and WC-1. $P$-value comparison between the half-lives of total WC-1 and nuclear FRQ is 0.009 , and between total WC-1 and nuclear $\mathrm{WC}-1$ is 0.007 . There is no significant difference between the half-lives of nuclear FRQ and nuclear WC-1 $(P=0.99)$. All $P$ values are adjusted by the Tukey method. $(C) \mathrm{WC}-1_{\mathrm{n}}$ is stable in the nucleus in the $f r q^{10}$ mutant. In the absence of FRQ, WC- $1_{n}$ stays in the nucleus even after CHX treatment. (D) In the fr $q^{9}$;MYC- $f r q 1$ mutant, FRQ is phosphorylated but does not turn over and WC-1 is stable as in $f r q^{10}$. The $f_{r} q^{9}$; MYC- $f_{r q 1}$ mutant lacks the coiled-coil domain of FRQ and cannot bind to the WCC (Cheng et al. 2001a). 
ylated (Fig. 3D) but fails to turn over in the normal manner. Total WC-1 levels are greatly reduced, probably because the stabilization of WC-1 mediated by hyperphosphorylated FRQ (Schafmeier et al. 2006) is absent. Interestingly, however, in this mutant, WC- $1_{n}$ appears as stably in the nucleus as it does in the frq-null mutant $\left(f r q^{10}\right)$ in CHX (Fig. 3, cf. C and D). Therefore, the physical interaction between FRQ and WC-1 seems to be important for the FRQ-dependent clearance of WC-1 from the nucleus. Based on these data, we propose that the FRQ-dependent clearance of the WC-1 from the nucleus is a closure mechanism of the negative feedback loop.

\section{The amount of the $F R Q_{n}: W C C_{n}$ complex oscillates}

Previous studies based principally on the examination of total cell extracts had shown that the vast majority of WCC is not associated with FRQ and led to the prediction that FRQ:WCC interactions were weak and/or transient (Schafmeier et al. 2005). However, the marked differential compartmentalization of FRQ and WC-1 makes it very difficult to accurately assess the amount of complex from analysis of whole-cell extracts. A number of studies have shown that most of FRQ is in the cytoplasm, whereas most of WC-1 is in the nucleus. Given this, unless the efficiency of a coimmunoprecipitation (co-IP) is close to $100 \%$, a co-IP of total extract with anti-FRQ will follow cytoplasmic FRQ and with antiWC-1 will follow predominantly nuclear WC-1, in both cases under-estimating the amount of FRQ:WCC simply due to mass action. To re-examine this issue, we performed a FRQ co-IP experiment using extracts from isolated nuclei, since FRQ is limiting in the nucleus, and compared this with similar analyses from total cell extracts (Fig. 4A). In agreement with previous work (Schafmeier et al. 2005), the co-IP data indicate that the percentage of WCC in complex with FRQ from the total lysates is between $1 \%$ and $3 \%$; it does not oscillate (Fig. 4B). In contrast, the co-IP results from the nuclear lysates show that an appreciable amount of WC-1 is complexed with FRQ - as much as $10 \%$ of the nuclear WC-1-and furthermore that the amount of $\mathrm{FRQ}_{\mathrm{n}}: \mathrm{WCC}_{\mathrm{n}}$ complex oscillates (Fig. 4B). This oscillation in the amount of the FRQ:WCC complex has also been confirmed recently by mass spectrometry analysis using epitope-tagged proteins (C. Baker, A. Kettenbach, J. Loros, S. Gerber, and J.C. Dunlap, in prep.). Figure 4, B and C, plots the amount of complex detected as a function of time, showing a very low and relatively constant amount as assessed from coIP from total cell extracts as compared with a clearly evident and cyclical amount detectable in co-IPs from nuclear extracts. Notably and as predicted (Hong et al. 2008), the level of $F R Q_{n}: W C-1_{n}$ complex is high around CT1 and low around CT10, and the peak of the complex is followed by the peak of $F_{R Q}$.

\section{Discussion}

A time-delayed negative feedback loop is at the core of the circadian rhythm's blueprint in various organisms,

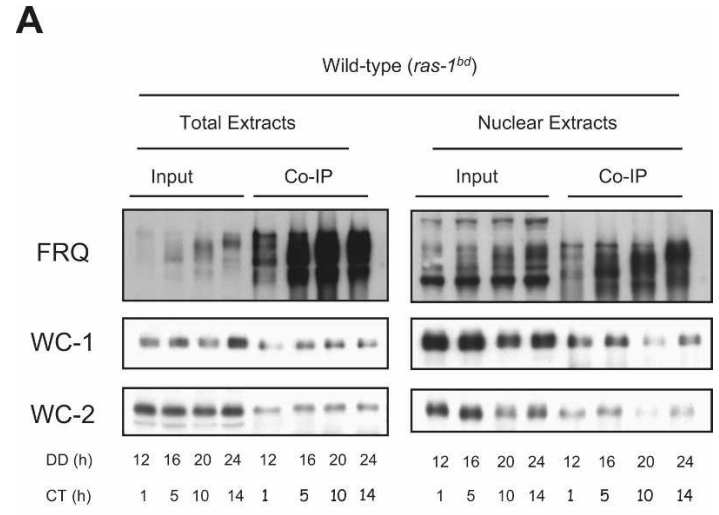

B

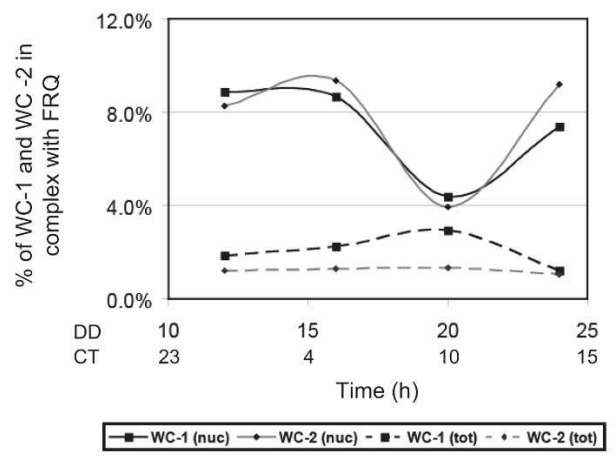

C

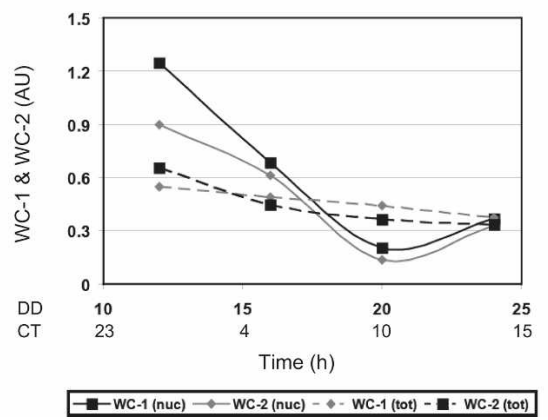

Figure 4. The level of the FRQ:WCC complex in the nucleus oscillates and does not simply reflect the nuclear level of FRQ. (A) Co-IP experiments with total lysates (left panels) versus nuclear lysates (right panels). FRQ polyclonal antibody was added to $\sim 1 \mathrm{mg}$ of protein for total lysates and $15 \mu \mathrm{g}$ for nuclear lysates. Total lysate blots represent $330 \mu \mathrm{g}$ of the immunoprecipitate and nuclear extract blots $7.5 \mu \mathrm{g}$ of protein. Ten micrograms of protein were loaded for total input and $1.5 \mu \mathrm{g}$ of protein for nuclear input. FRQ and WC-1 are detected from replicate blots and WC-2 from the same blot as WC-1. FRQ is bound to the WCC at all times. The oscillation of the FRQ:WCC complex is reflected in the amount of WC-1 and WC-2 that coimmunoprecipitates with FRQ. This is apparent in the nuclear lysates whereas in the total lysates the amount of WC-1 and WC-2 simply reflect the amount of FRQ. $(B)$ The densitometic analysis shown in $A$ indicates a cycle between $\sim 4 \%$ and $9 \%$ of the WCC in complex with FRQ in the nucleus. The amount of coimmunoprecipitated WCC is here normalized to the input WC-1. $(C)$ As in $B$, but the amount of coimmunoprecipitated WCC is normalized to the amount of immunoprecipitated FRQ. 
including Neurospora. FRQ inhibits its own activator (Aronson et al. 1994), the heterodimeric transcription factor WCC (Crosthwaite et al. 1997), with which it interacts (Cheng et al. 2001a, 2002; Denault et al. 2001), but the nature of this inhibition has remained the object of much study. The first and simplest model, based on the demonstrated interactions among the component parts, was simply that FRQ would bind to the WCC and reduce its activity. This notion gained some support from studies describing a rhythm in the ability of WCC from whole-cell extracts to bind to DNA, studies in which it was shown that addition of in vitro translated FRQ reduced the ability of in vitro translated WCC to bind to DNA (Froehlich et al. 2003). However, more careful estimations of intracellular distributions of FRQ and the WCC showed FRQ to be generally cytoplasmic and WCC nuclear to such an extent that the simple model seemed untenable; the simultaneous discovery that phosphorylation of WCC reduced its ability to bind to DNA led to the hypothesis that FRQ-induced phosphorylation of WCC was the basis of negative feedback ( He and Liu 2005; Schafmeier et al. 2005). Based as it is on extrapolations from measurements of cytoplasmic and nuclear FRQ levels, this later intuitive model assumes no dynamic shuttling of proteins between compartments. In hindsight, the role of FRQ-promoted phosphorylation in modulating the affinity of WCC for DNA may explain the results of Froehlich et al. (2003), too, in that the in vitro translated FRQ could have acted to promote phosphorylation of the in vitro translated WCC by the casein kinase 1 (Basu et al. 2003) and casein kinase 2 (Majumdar et al. 2002) that are known to be present in the reticulocyte lysates used for the in vitro translations.

Two separate issues conspired to suggest that further examination of the mechanism of negative feedback would be informative. In the first, development of a mathematical model (Hong et al. 2008) of the oscillator revealed that the original stoichiometric model based on FRQ-WCC interactions could support oscillations in WCC activity and frq expression, especially if the assumption of a static population of FRQ within the nucleus was questioned and shuttling of FRQ from the cytoplasm into and out of the nucleus was allowed. The second spur was the realization that, given the relative inefficiency of co-IPs, measurements from whole-cell extracts based on co-IP data could never accurately report levels of a FRQ:WCC complex, especially a nuclear complex. Based on this, rhythmic FRQ-dependent clearance of WC-1 from the nucleus was postulated as a mechanism for closure of the circadian feedback loop, and the data presented here are all consistent with this hypothesis. In particular, FRQ-dependent clearance of WC-1 may be satisfied by two different possibilities: the rapid degradation of the FRQ:WC-1 complex in the cytoplasm (or in the nucleus) (Fig. 5A), or rapid export (shuttling) of the FRQ:WC-1 complex from the nucleus (Fig. 5B). Both scenarios would generate robust oscillations that fit the known molecular profiles. Because our in silico experiments (Supplemental Material) indicate that either catalytic or noncatalytic functions of FRQ can generate robust oscillatory systems, it seems premature to suggest that the mechanism of negative feedback is exclusively through either mechanism. Rather, it becomes clear that both (catalytic and noncatalytic) scenarios are plausible based on mathematical modeling so long as there exists FRQ-dependent clearance of WC-1 from the nucleus.

Indeed, such FRQ-dependent clearance of WC-1 has been experimentally validated (Fig. 3A,C) and seemed to require physical interactions between FRQ and WC-1 (Fig. 3D). A finding not predicted from the model was the rediscovery (after Lee et al. 2000) of WC-1 turnover correlated with the appearance of hypophosphorylated FRQ (Fig. 2A,B). At first glance, it would appear that this could also contribute to the FRQ-mediated reduction of
A

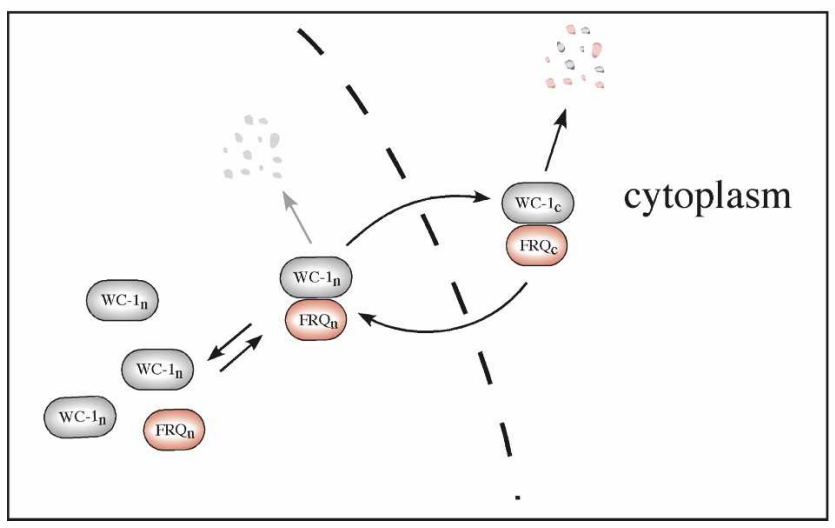

B

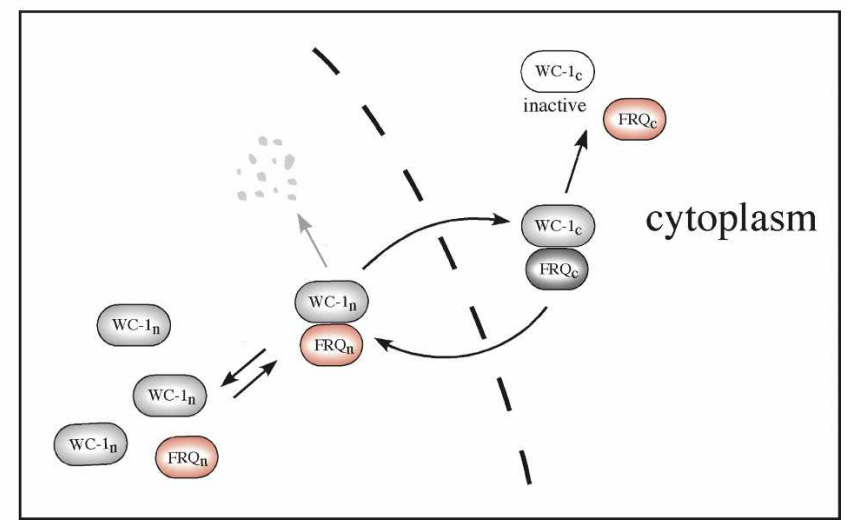

Figure 5. Models for FRQ-dependent clearance of WC-1 from the nucleus. $(A)$ Model with rapid shuttling of FRQ ${ }_{n}:$ WC- $1_{n}$ accompanied by turnover in the cytoplasm. $(B)$ Model with rapid shuttling of $\mathrm{FRQ}_{\mathrm{n}}$ :WC- $1_{\mathrm{n}}$ accompanied by the inactivation of WC-1 in the cytoplasm. FRQ is recycled (equivalent to a "catalytic-like" interaction between FRQ and WCC). Gray arrow indicates that the reaction rate reflecting nuclear degradation is set to 0 . Given a pool of cytoplasmic FRQ and allowing shuttling, mathematical analysis of both models (as described in the Supplemental Material) shows each capable of generating robust oscillations. The detailed wiring diagram of the complete model with equations is described in the Supplemental Material. (Figures adapted from Hong et al. 2008 with permission from the Biophysical Society.) 
WCC actively, perhaps serving initially and acutely to turn down frq expression while FRQ levels climb. However, the FRQ-dependent clearance of WC-1 from the nucleus indicates that clearing of WC-1 from the nucleus is a means of closing the circadian negative feedback loop. This can be achieved by two ways: (1) degradation of the FRQ:WCC complex in the nucleus, and/or (2) FRQ-dependent export of WC-1 from the nucleus. If it is FRQ-dependent degradation of WC-1 in the nucleus that results in FRQ-dependent clearance of WC-1 from the nucleus, that should be reflected in the total lysates considering that most WC-1 is in the nucleus. However, the obvious differences in WC-1 dynamics in the total versus nuclear lysates clearly signify FRQ-dependent export of WC-1 from the nucleus and support the hypothesis that nucleocytoplasmic shuttling of the WCC is a means of closing the circadian negative feedback loop.

In summary, there appear to be four ways in which FRQ influences the level and activity of the WCC, through hypophosphorylated FRQ-mediated turnover of WC-1 (Fig. 2; Lee et al. 2000), through hyperphosphorylated FRQ-mediated accumulation/stabilization of FRQ (Lee et al. 2000; Schafmeier et al. 2006), though FRQpromoted phosphorylation of WCC ( He and Liu 2005; Schafmeier et al. 2005), and through formation of FRQ:WCC complexes followed by FRQ-mediated clearance of WCC (Figs. 3, 4). To varying degrees, these activities are echoed among the regulatory loops described for animal clocks (for review, see Mackey 2007). The first, FRQ-mediated turnover of WC-1, finds a precedent in the observation that in embryonic mouse fibroblasts, robust transcription of Per2 is tightly associated with massive degradation of the CLOCK/BMAL1 heterodimer (Kwon et al. 2006). In the interlocked feedback loops of the mammalian clock, the negative element PER2 acts through Rev-ERB $\alpha$ to promote increases in BMAL1 expression in a mechanism, producing the same result as the FRQ-promoted stabilization of WC-1 (Shearman et al. 2000; Preitner et al. 2002). Third, as is the case with the WCC, CLOCK and BMAL1 undergo robust circadian changes in phosphorylation that are believed to influence their activity (Lee et al. 2001; for review, see Virshup et al. 2007). Finally, the FRQ-influenced clearance of WCC described here as playing a major role in closure of the circadian feedback loop finds a precedent in the nucleocytoplasmic shuttling of the transcription factor, BMAL1, observed in mammalian systems (Tamaru et al. 2003; Kwon et al. 2006). In the above two studies, the investigators show clock-controlled shuttling of BMAL1, although whether it is PER or CRY dependent is still undetermined. Collectively, then, the data suggest that there may be similar mechanistic details for closing the circadian negative feedback loop from Neurospora to mammals.

\section{Materials and methods}

Strains and growth conditions

Strains used for the experiments were clock wild-type 328-4 (ras-1 ${ }^{b d}$; A), 164-273::pBA50 (ras- ${ }^{b d}$;fr ${ }^{10}{ }^{10} q a-2 p-f r q($ his-3::frq), a), 358-6 $\left(\right.$ ras- $1^{\text {bd }} ;$ frq $^{10}$, a), and $f r q^{9} ;$ MYC-frq1. Liquid culture

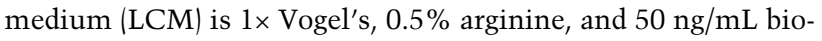
tin with glucose at $0.1 \%$ or $2 \%$ as a carbon source. For total lysates, samples were grown in the LCM and harvested as described previously (Belden et al. 2007).

\section{DD-to-CT conversions}

DD indicates the number of hours that a culture was held in darkness, after a transfer from LL, prior to sampling. CT represents subjective time within the daily circadian cycle when the clock is running under DD. By convention, one circadian cycle $(-22 \mathrm{~h}$ in Neurospora) is broken into 24 circadian hours, and CT12 reflects the light-to-dark transition. Thus in DD, subjective day is CT0-12 and subjective night is CT12-24.

\section{CHX experiments and measurements of half-life}

Mycelial pads were grown in $50 \mathrm{~mL}$ of $2 \%$ glucose LCM in 250-mL Erlenmeyer flasks with overnight shaking at $25^{\circ} \mathrm{C}$ in $\mathrm{LL}$ before transfer to $\mathrm{DD}$ at $25^{\circ} \mathrm{C}$. Samples were treated with 40 $\mu \mathrm{g} / \mathrm{mL} \mathrm{CHX}$ at indicated DD times and harvested over $12 \mathrm{~h}$ with 4-h resolution. Half-lives were measured as in Ruoff et al. (2005): Degradation data of FRQ and WC-1 were used to calculate degradation rates of each protein $\left(k_{i}\right)$ by fitting data to firstorder decay kinetics [i.e., $\mathrm{P}=\mathrm{P}_{0} \times \exp \left(-k_{i} \mathrm{t}\right)$, where $\mathrm{P}$ is protein (i.e., FRQ)], and the degradation rate is used to calculate half-life $\left(t_{1 / 2}=\ln 2 / k_{i}\right)$.

\section{QA-induction experiments}

Mycelial pads were grown in $50 \mathrm{~mL}$ of $2 \%$ glucose LCM in 250-mL Erlenmeyer flasks with overnight shaking at $25^{\circ} \mathrm{C}$ in LL. Samples were then transferred to $50 \mathrm{~mL}$ of $0.1 \%$ glucose LCM and grown overnight at $25^{\circ} \mathrm{C}$ in LL before adding QA for efficient induction. QA $\left(10^{-2} \mathrm{M}\right)$ was applied to samples at the time of transfer from LL to DD. Samples were harvested at indicated DD times as described previously (Belden et al. 2007). For QA induction, wash, and QA reinduction experiment, samples were treated as above except that they were transferred yet again from QA-containing $0.1 \%$ glucose LCM to fresh $0.1 \%$ glucose LCM $(50 \mathrm{~mL})$. Samples were retreated with QA $\left(10^{-2} \mathrm{M}\right)$ and harvested at indicated DD times. For the QA induction and CHX experiment, samples were grown as above except that the CHX was applied after either 4-h or 12-h induction, and harvested over $12 \mathrm{~h}$ in the CHX with 4-h resolution.

\section{Isolation of nuclei}

Nuclei were isolated with a protocol modified from that published previously (Loros and Dunlap 1991; Luo et al. 1998). The mycelial pads were grown in $350 \mathrm{~mL}$ of $2 \%$ glucose LCM in 1-L Erlenmeyer flasks overnight at $25^{\circ} \mathrm{C}$ in LL before transferring to DD. CHX was added at indicated DD times, and samples were harvested at indicated DD times. The mycelial pads (5-8 g, blotdried wet weight) were ground with $\sim 12 \mathrm{~g}$ of cold glass beads in a bead-beater (Biospec Products) containing $15 \mathrm{~mL}$ of cold buffer A (1 M sorbitol, 7\% [w/v] Ficoll, 20\% [v/v] glycerol, $5 \mathrm{mM}$ magnesium acetate, $3 \mathrm{mM} \mathrm{CaCl}, 3 \mathrm{mM}$ dithiothreitol [DTT], $50 \mathrm{mM}$ Tris- $\mathrm{HCl}$ at $\mathrm{pH} 7.5$ ). The crude homogenate was filtered through cheesecloth, and 2 vol of cold buffer B $(10 \%[\mathrm{v} / \mathrm{v}]$ glycerol, $5 \mathrm{mM}$ magnesium acetate, $25 \mathrm{mM}$ Tris- $\mathrm{HCl}$ at $\mathrm{pH} 7.5$ ) were slowly added with stirring. The diluted homogenate was centrifuged at $3000 \mathrm{~g}$ for $7 \mathrm{~min}$ at $4^{\circ} \mathrm{C}$ in a SW 28 rotor to remove cell debris. The resulting supernatants were removed (this is referred as the total cell lysate), layered onto 5 -mL step gradi- 
ents ( $1 \mathrm{M}$ sucrose, $10 \%$ [v/v] glycerol, $5 \mathrm{mM}$ magnesium acetate, $1 \mathrm{mM}$ DTT, $25 \mathrm{mM}$ Tris- $\mathrm{HCl}$ at $\mathrm{pH} 7.5$ ) in a $50-\mathrm{mL}$ tube and centrifuged at $9400 \mathrm{~g}$ for $15 \mathrm{~min}$ at $4^{\circ} \mathrm{C}$ in a SW 28 rotor to pellet the nuclei. The resulting nuclear pellets were gently lysed in RIPA (150 mM sodium chloride, 1\% NP-40, 0.5\% sodium deoxycholate, $0.1 \%$ SDS, $50 \mathrm{mM}$ Tris at $\mathrm{pH} 7.5$ ) buffer and centrifuged at $13,2000 \mathrm{rpm}$ for $15 \mathrm{~min}$ at $4^{\circ} \mathrm{C}$. All the buffers contain $1 \mu \mathrm{g} / \mathrm{mL}$ leupeptin, $1 \mu \mathrm{g} / \mathrm{mL}$ pepstatin $\mathrm{A}$, and $1 \mathrm{mM}$ phenylmethylsulfonyl fluoride (PMSF). Prior to the nuclear extraction, a portion of mycelial tissue is saved for a total lysis.

\section{Co-IP and Western blots}

Co-IP experiments with the total lysates were performed as described previously except that $1 \%$ Triton was omitted during the wash (Denault et al. 2001). Co-IP experiments with the nuclear extracts were performed as with total lysates except that $15 \mu \mathrm{g}$ of protein were used instead of $1 \mathrm{mg}$. Immunoblot analysis was done as described (Garceau et al. 1997) with the FRQ, WC-1, or WC-2 antibodies as described in previous publications (Garceau et al. 1997; Lee et al. 2000; Denault et al. 2001).

\section{Acknowledgments}

We thank Yi Liu for providing the strain $f r q^{9}$; MYC-frq1. We also thank Hildur Colot and John Tyson for helpful comments, and Zhongze Li in the Biostatistics Core at Dartmouth Medical School for statistical analysis of the densitomeric data from the Western blots. The preliminary draft of this manuscript was initiated at the KITP at the UCSB during Biological Switches and Clocks workshop. This work was supported by grants from the National Institutes of Health to J.C.D. (GM34985 and GM68087) and to J.C.D. and J.J.L. (GM08336), and the National Academies Keck Futures Initiative Signaling Grant to C.I.H.

\section{References}

Akman, O.E., Locke, J.C., Tang, S., Carre, I., Millar, A.J., and Rand, D.A. 2008. Isoform switching facilitates period control in the Neurospora crassa circadian clock. Mol. Syst. Biol. 4: 186. doi: 10.1038/msb.2008.5.

Aronson, B.D., Johnson, K.A., Loros, J.J., and Dunlap, J. 1994. Negative feedback defining a circadian clock: Autoregulation of the clock gene frequency. Science 263: 1578-1584.

Basu, U., Si, K., Deng, H., and Maitra, U. 2003. Phosphorylation of mammalian eukaryotic translation initiation factor 6 and its Saccharomyces cerevisiae homologue Tif6p: Evidence that phosphorylation of Tif6p regulates its nucleocytoplasmic distribution and is required for yeast cell growth. Mol. Cell. Biol. 23: 6187-6199.

Belden, W.J., Loros, J.J., and Dunlap, J.C. 2007. Execution of the circadian negative feedback loop in Neurospora requires the ATP-dependent chromatin-remodeling enzyme CLOCKSWITCH. Mol. Cell 25: 587-600.

Cheng, P., Yang, Y., Heintzen, C., and Liu, Y. 2001a. Coiled-coil domain-mediated FRQ-FRQ interaction is essential for its circadian clock function in Neurospora. EMBO J. 20: 101108.

Cheng, P., Yang, Y., and Liu, Y. 2001b. Interlocked feedback loops contribute to the robustness of the Neurospora circadian clock. Proc. Nat1. Acad. Sci. 98: 7408-7413.

Cheng, P., Yang, Y., Gardner, K.H., and Liu, Y. 2002. PAS domain-mediated WC-1/WC-2 interaction is essential for maintaining the steady-state level of $\mathrm{WC}-1$ and the function of both proteins in circadian clock and light response of Neurospora. Mol. Cell. Biol. 22: 517-524.

Crosthwaite, S.K., Dunlap, J.C., and Loros, J.J. 1997. Neurospora wc-1 and wc-2: Transcription, photoresponses, and the origins of circadian rhythmicity. Science 276: 763-769.

Denault, D.L., Loros, J.J., and Dunlap, J.C. 2001. WC-2 mediates WC-1-FRQ interaction within the PAS protein-linked circadian feedback loop of Neurospora. EMBO J. 20: 109-117.

Dunlap, J.C. 1999. Molecular bases for circadian clocks. Cell 96: 271-290.

Froehlich, A.C., Loros, J., and Dunlap, J.C. 2003. Rhythmic binding of a WHITE COLLAR-containing complex to the frequency promoter is inhibited by FREQUENCY. Proc. Natl. Acad. Sci. 100: 5914-5919.

Gallego, M., Eide, E.J., Woolf, M.F., Virshup, D.M., and Forger, D.B. 2006. An opposite role for tau in circadian rhythms revealed by mathematical modeling. Proc. Natl. Acad. Sci. 103: $10618-10623$.

Garceau, N.Y., Liu, Y., Loros, J.J., and Dunlap, J. 1997. Alternative initiation of translation and time-specific phosphorylation yield multiple forms of the essential clock protein FREQUENCY. Cell 89: 469-476.

$\mathrm{He}$, Q. and Liu, Y. 2005. Molecular mechanism of light responses in Neurospora: From light-induced transcription to photoadaptation. Genes \& Dev. 19: 2888-2899.

He, Q., Cheng, P., Yang, Y., He, Q., Yu, H., and Liu, Y. 2003. FWD1-mediated degradation of FREQUENCY in Neurospora establishes a conserved mechanism for circadian clock regulation. $E M B O J$. 22: 4421-4430.

Hong, C.I., Conrad, E.D., and Tyson, J.J. 2007. A proposal for robust temperature compensation of circadian rhythms. Proc. Natl. Acad. Sci. 104: 1195-1200.

Hong, C.I., Jolma, I.W., Loros, J.J., Dunlap, J.C., and Ruoff, P. 2008. Simulating dark expressions and interactions of FRQ and WC-1 in the Neurospora circadian clock. Biophys. J. 94: 1221-1232.

Kwon, I., Lee, J., Chang, S.H., Jung, N.C., Lee, B.J., Son, G.H., Kim, K., and Lee, K.H. 2006. BMAL1 shuttling controls transactivation and degradation of the CLOCK/BMAL1 heterodimer. Mol. Cell. Biol. 26: 7318-7330.

Lee, K., Loros, J.J., and Dunlap, J.C. 2000. Interconnected feedback loops in the Neurospora circadian system. Science 289: $107-110$.

Lee, C., Etchegaray, J., Cagampang, F.R.A., Loudon, A.S.I., and Reppert, S.M. 2001. Posttranslational mechanisms regulate the mammalian circadian clock. Cell 107: 855-867.

Liu, Y. and Bell-Pedersen, D. 2006. Circadian rhythms in Neurospora crassa and other filamentous fungi. Eukaryot. Cell 5: 1184-1193.

Locke, J.C., Kozma-Bognar, L., Gould, P.D., Feher, B., Kevei, E., Nagy, F., Turner, M.S., Hall, A., and Millar, A.J. 2006. Experimental validation of a predicted feedback loop in the multi-oscillator clock of Arabidopsis thaliana. Mol. Syst. Biol. 2: 59. doi: 10.1038/msb4100102.

Loros, J.J. and Dunlap, J.C. 1991. Neurospora crassa clock-controlled genes are regulated at the level of transcription. Mol. Cell. Biol. 11: 558-563.

Luo, C., Loros, J.J., and Dunlap, J. 1998. Nuclear localization is required for function for the essential clock protein FRQ. EMBO I. 17: 1228-1235.

Mackey, S.R. 2007. Biological rhythms workshop IA: Molecular basis of rhythms generation. Cold Spring Harb. Symp. Quant. Biol. 72: 7-19.

Majumdar, R., Bandyopadhyay, A., Deng, H., and Maitra, U. 2002. Phosphorylation of mammalian translation initiation factor 5 (eIF5) in vitro and in vivo. Nucleic Acids Res. 30: 
Hong et al.

$1154-1162$.

Preitner, N., Damiola, F., Lopez-Molina, L., Zakany, J., Duboule, D., Albrecht, U., and Schibler, U. 2002. The orphan nuclear receptor REV-ERB $\alpha$ controls circadian transcription within the positive limb of the mammalian circadian oscillator. Cell 110: 251-260.

Ruoff, P., Loros, J.J., and Dunlap, J.C. 2005. The relationship between FRQ-protein stability and temperature compensation in the Neurospora circadian clock. Proc. Natl. Acad. Sci. 102: 17681-17686.

Schafmeier, T., Haase, A., Kaldi, K., Scholz, J., Fuchs, M., and Brunner, M. 2005. Transcriptional feedback of Neurospora circadian clock gene by phosphorylation-dependent inactivation of its transcription factor. Cell 122: 235-246.

Schafmeier, T., Kaldi, K., Diernfellner, A., Mohr, C., and Brunner, M. 2006. Phosphorylation-dependent maturation of Neurospora circadian clock protein from a nuclear repressor toward a cytoplasmic activator. Genes \& Dev. 20: 297-306.

Shearman, L.P., Sriram, S., Weaver, D.R., Maywood, E.S., Chaves, I., Zheng, B., Kume, K., Lee, C.C., van der Horst, G.T., Hastings, M.H., et al. 2000. Interacting molecular loops in the mammalian circadian clock. Science 288: 10131019.

Talora, C., Franchi, L., Linden, H., Ballario, P., and Macino, G. 1999. Role of a White Collar-1-White Collar-2 complex in blue-light signal transduction. EMBO J. 18: 4961-4968.

Tamaru, T., Isojima, Y., van der Horst, G.T., Takei, K., Nagai, K., and Takamatsu, K. 2003. Nucleocytoplasmic shuttling and phosphorylation of BMAL1 are regulated by circadian clock in cultured fibroblasts. Genes Cells 8: 973-983.

Ueda, H.R. 2007. Systems biology of mammalian circadian clocks. Cold Spring Harb. Symp. Quant. Biol. 72: 365-380.

Virshup, D.M., Eide, E.J., Forger, D.B., Gallego, M., and Harnish, E.V. 2007. Reversible protein phosphorylation regulates circadian rhythms. Cold Spring Harb. Symp. Quant. Biol. 72: 413-420. 


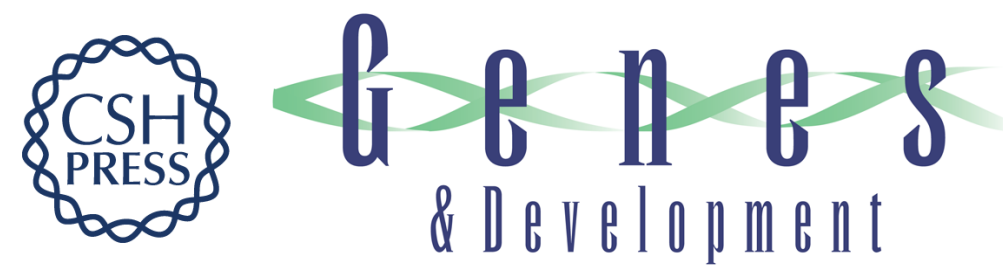

\section{Closing the circadian negative feedback loop: FRQ-dependent clearance of WC-1 from the nucleus}

Christian I. Hong, Peter Ruoff, Jennifer J. Loros, et al.

Genes Dev. 2008, 22: originally published online November 7, 2008

Access the most recent version at doi:10.1101/gad.1706908

\section{Supplemental http://genesdev.cshlp.org/content/suppl/2008/11/06/gad.1706908.DC1 Material}

References This article cites 35 articles, 26 of which can be accessed free at: http://genesdev.cshlp.org/content/22/22/3196.full.html\#ref-list-1

\section{License}

Email Alerting

Receive free email alerts when new articles cite this article - sign up in the box at the top Service

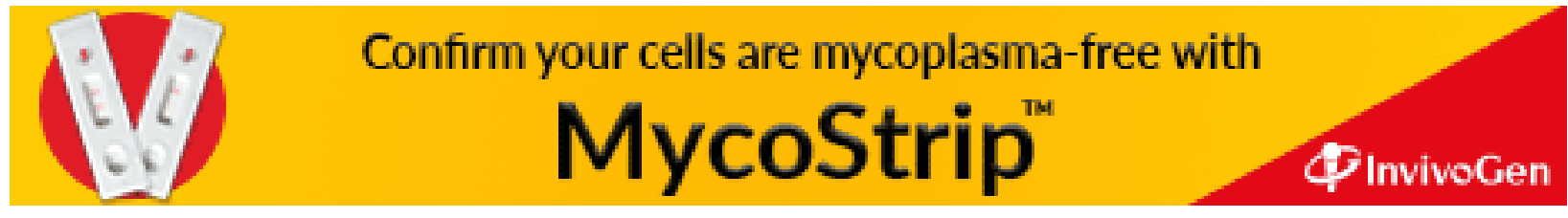

